\title{
An Assessment of DInSAR Potential for Simulating Geological Subsurface Structure
}

\author{
$\underline{\text { N.F. Moghaddam }}^{\text {a }}$, C. Rüdiger ${ }^{\text {a }}$, S. Samsonov ${ }^{\text {, }}$ J.P.Walker ${ }^{\text {a }}$, M. Hall ${ }^{\text {b }}$ \\ ${ }^{a}$ Department of Civil Engineering, Monash University, Victoria, Australia \\ Email: negin.moghaddam@monash.edu.au \\ ${ }^{b}$ School of Geosciences, Monash University, Victoria, Australia \\ ${ }^{c}$ Natural Resources Canada, Ottawa, Canada
}

\begin{abstract}
Knowledge of the subsurface properties driving subsidence is of key importance in conducting geophysical surveys such as self-potential, magneto telluric, and reflection seismic, as this can help defining the areas with downward water flow, collapsed features at depth, or overburden affected regions. However, accurate and affordable subsurface information is difficult to obtain with currently available field technology, because changes occur on the time scale of years which means monitoring must be undertaken over extended time periods. Consequently, data are typically limited to borehole logs, well production data, seismic tests, or point subsidence measurements. As these data are laborious to collect, and spatially / temporally sparse, processing Synthetic Aperture Radar (SAR) remote sensing data can provide accurate surface deformation information, which is spatially and temporally dense, allowing to define areas susceptible to subsidence.
\end{abstract}

To enhance the understanding of the subsurface structure from surface deformation monitoring, a methodology needs to be developed to relate the subsurface data to the high resolution time-lapse remote sensing data in a consistent and regular manner. An accepted technique for monitoring surface deformations is interferometric SAR remote sensing. Here, this approach is applied within the Surat Basin, Australia, to demonstrate its potential to determine the geological subsurface structure. Over the past two- decades, studies in the field of interferometric SAR analysis have focused mainly on generating elevation maps or maps of the deformation induced by unknown underground processes. This research will address the development of a methodology to predict subsurface structural geometries/distribution from surface deformation data, aiming to identify hidden/blind faults or hydraulic barriers using long-term (time lapse) surface observations, as an innovative and cost-effective tool for understanding the subsurface. It is expected that this approach will be able to extend spatially or temporally sparse in-situ datasets, predict subsurface hazards such as sinkholes, and identify where to acquire more in-situ data, thus mitigating risk in resource management.

There are three main focus areas in this research: the monitoring of surface deformation, the simulation of the subsurface structure, and the retrieval of the required subsurface parameters through the use of remotely sensed surface observations to improve the available structural models. To achieve these goals, as an initial step the areas which are susceptible to surface deformation due to ground water extraction are pinpointed using Multidimensional Small Baseline Subset (MSBAS) technique for interferometric pairs of C-, L- and Xband SAR datasets. These observations identify the potential regions for any geological boundaries such as faults or changes in the formation sequence which may have an impact on observed vertical and horizontal deformation signals. For the next stage, it is proposed that these observations could be applied for geophysical inverse modelling to assess the temporal behaviour and impact of boundaries for the observed deformation. By estimating the best parameter space through inverse modelling, it is hypothesized that the previously available subsurface structural model provided by integration of multiple geophysical datasets like seismic, gravity, radiometric and magnetic, may be improved in conjunction with SAR interferometry.

For the Surat Basin, preliminary DInSAR results (ERS-Envisat C-band; 1992 to 2005) show considerable deformation patterns in 2004-2005. These signals are still present in the 2012-2013 interferograms and are coincident with regional underground activities, confirming the anthropogenic impact on subsidence. While this is a promising development, accurate and long term measurement of deformation using such sparse satellite dataset is still difficult to achieve and a more advanced DInSAR processing algorithm for integrating various space-borne SAR data with different acquisition parameters (i.e. temporal sampling, spatial resolution, wave-band and polarization, etc.) is required. This will improve the temporal and spatial resolution of the interferograms. In this study, first results of the DInSAR processing chain over the Surat Basin, including a discussion on the optimization scheme layout are proposed for future use.

Keywords: Differential Interferometric SAR (DInSAR), subsurface structural modeling, ground water withdrawal, subsidence 


\section{INTRODUCTION}

Due to the exploration of earth resources, farming and infrastructure, there is a significant need to manage the risk to critical resources, in particular water within subsurface aquifers. The management of such resources is not being completed and could be defined by assigning confidence to the presence, distribution and composition of seals (shales) and aquifers (sandstones) over time. Risk is a difficult concept to define, with data sets at different scales and obtained with various methods, coming from different sources. The critical problem is how to integrate these different data sources, so as to interpret the available subsurface data appropriately and thus manage subsurface water resources. While geophysical measurements may be very precise (Anderssohn et al., 2009), there are several model/parameter combinations which could equally fit to a given data set, meaning that the interpretation can be inaccurate if other site information such as borehole logs are not taken into account. Moreover, these measurements are very costly and time consuming, especially in the planning stage, and provide only localized information. However, geophysics measurements can identify geological and other sub-surface features which may pose problems during construction if not accounted for planning and design stages. Thus, it is important to choose an appropriate investigation strategy based on the type of target, its physical and chemical properties, and the extent of monitoring area for suitable geophysical surveys.

Differential Interferometric SAR (DInSAR) (Massonnet \& Feigl, 1998) has been improved over the past two decades, showing a clear potential in mapping of long-term surface deformation resulting from changes in volume, pressure and temperature after underground reservoir extraction or seismic events. In most displacement cases, such as earthquakes, subsidence, and landslides, it has been claimed that the deformation patterns on the surface are controlled by subsurface structures like faults or hydraulic barriers (Zhang, 2010). However, none of them have used DInSAR extracted elevation maps to define the subsurface structures in the way that geophysics testing does.

Consequently, the scope of this research is to develop a method for retrieving subsurface structuring and identify hidden faults/hydraulic barriers using DInSAR surface observations in combination with geophysical modeling. The Surat Basin in Queensland is used as a case study in this paper to verify the potential of DInSAR as a valuable and cost-effective reconnaissance exploration tool for planning seismic acquisition programs. Therefore, traditional DInSAR processing for selected SAR images with temporal and spatial baselines smaller than the required thresholds first needs to be undertaken. These deformation maps are then used as an input for geophysical inverse modeling to derive a suitable estimated subsurface model, allowing a better retrieval of the properties of shallow depth geological structures.

In this paper, preliminary results of C-band data obtained with the European Space Agency's (ESA) ERS-1 SAR and Envisat ASAR imagery processing with DInSAR technique are presented. The deformation signals covering the Eastern part of the Surat Basin from 1992 to 2005 are cross-checked with RADARSAT-2 observations for the period 2012-2013, confirming a long-term gradual development in ground displacement.

\section{THE SURAT BASIN}

The Surat Basin (Figure 1) is a geological basin in Eastern Australia with Jurassic through to Cretaceous aged sediments derived from Triassic and Permian age rocks. Surat has been traditionally known for its agricultural fields and significant underground resources. Whilst most of the region has been extensively cleared for agriculture, there is not much in terms of vegetation in the area. Since 2000, there has been extensive ground water extraction for different applications which made it susceptible to groundwater level changes at depths of 300-600m (Moran \& Vink, 2010).

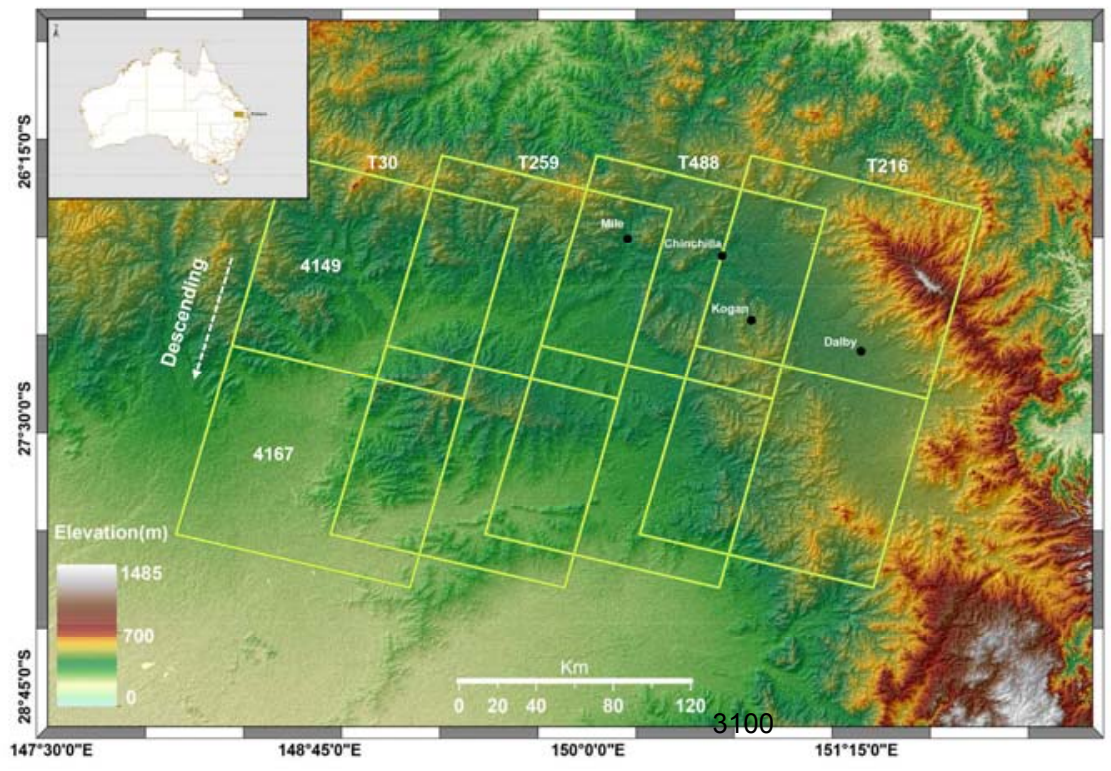

Figure 1. Envisat ASAR image mode coverage extent within the Surat Basin (2003-2005) with descending path and right-looking angle in two main 4149 and 4167 scenes for all tracks 


\section{DIFFERENTIAL INTERFEROMETRIC SAR (DINSAR)}

\subsection{Overview}

Differential Interferometric SAR (DInSAR) is a powerful and well-established remote sensing technique for highly accurate measurements of surface topography, ground deformation, and subsidence (Rosen et al., 2000). For a given scene, DInSAR (Figure 2) compares the phase of two or more complex radar images to achieve this. Since the phase of each pixel within a SAR image contains slant range information accurate to a fraction of the radar wavelength, any variation in the temporal baseline between interferometric acquisitions makes it possible to detect and measure small path length differences (slant range changes, or deformations) with accuracies in the order of sub-centimeters. The gradual advancement of DInSAR algorithms towards a multi-temporal SAR analysis allows the production of deformation time-series from a single set of SAR observations known as the Small Baseline Subset (SBAS) (Berardino et al., 2002), considering the spatial and temporal baseline as the critical threshold. In this research, direct integration of all available InSAR datasets using the Multidimensional Small Baseline Subset (MSBAS) methodology (Samsonov and d'Oreye, 2012) for producing 2D time-series of deformation will be used. Essentially, when two or more DInSAR data sets with overlapping temporal and spatial coverage are available, the signal's accuracy will be improved by this approach. The technique is based on the SBAS method, modified to account for variations in sensor parameters between the sensors.

\subsection{Satellite Dataset}

For monitoring purposes, ERS-1SAR and Envisat ASAR archived imagery for the descending tracks 30, 259, 488 and 216 for both 4149 and 4167 frames were obtained from ESA (Figure 1) for the period 1992-2010. First results obtained from SARPROZ software using an algorithm based on the DInSAR technique (Perissin et al., 2012), indicated that among those tracks, 488 and 216 contain deformation signals, confirming reported gas emissions (Queensland Water Commission, 2012). These deformation anomalies have also been verified using RADARSAT-2[only ascending mode is available], which operates at a similar wavelength, for two different scenes covering the Envisat footprints. In the future, through the acquisition of the Japan Aerospace Exploration Agency's (JAXA) ALOS L-band dataset (more than 50 images), the magnitude and spatial extent of any deformation signals in those parts of the Surat basin which may not be detected using Cband interferograms will be determined. Such problems may arise due to seasonal variations caused by expansion and contraction of the surface clays in response to changes in moisture.

\subsection{Preliminary DInSAR deformation maps}

The initial DInSAR processing results show that some of the interferograms did not produce sensible deformation maps due to the limited availability of the C-band acquisitions, as well as incoherent signals. In Figure 3, the probable deformation signal in the lower part of track 30 is not reliable as it only appears in one interferogram. Moreover, in the upper part of track 30 and lower part of track 488 atmospheric water vapor fluctuations during the observation period are likely the cause for the observed intermittent phase perturbation. Moreover, both scenes of track 259 and the lower scene of track 216 display signals similar to deformation signals. However, when checked against the other interferograms in this area, it was noticed that those signals are not persistent through time, which is a prerequisite to consider an observation as a deformation signal. In other words, significant changes in the spatial distribution of the deformation anomalies over time rule out a persistent subsidence event. On the contrary, within the overlay of Frame 4149 of tracks 488 and 216, three areas with temporally consistent deformation signals are identified. 


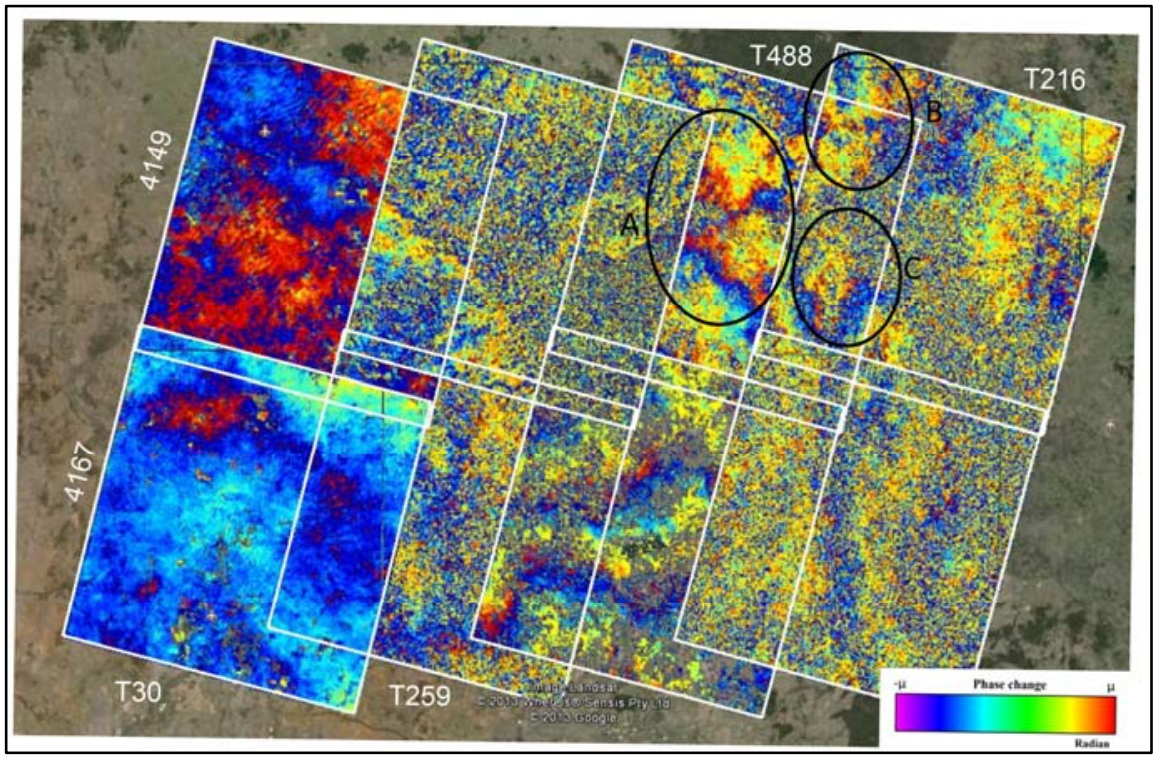

Figure 3. Preliminary DInSAR processing results of Envisat ASAR (2003-2005) in descending path. The main deformation areas(A,B, and C) are highlighted in Tracks 488, 216 both in 4149 frame.

For cross-validation of those findings, ERS-1 and RADARSAT-2 Cband imagery were processed for 19921995 (Figure 4) and 2012-2013 (Figure 5) respectively. In two ERS-1 observations, traces of deformation signals in the overlapping area (B and $\mathrm{C}$ ) are distinguishable. Two ascending scenes of RADARSAT-2 covering the proposed area with different viewing angle, confirm that the deformation in the region is still under progress. At this stage, the results presented in Figures 3-5 are preliminary deformation signals obtained using a conventional DInSAR technique. These deformation signals were obtained using more than ten interferograms and could be useful for subsurface structural modeling. However, to cover the monitoring gaps due to the sparse coverage of C-band satellites in this region, ALOS data, obtained at the shorter L-band wavelength, could be used alongside the C-band observations. For this purpose a number of ALOS data spanning from 2006 to 2011 are being obtained and processed for future analysis. Because of better signal penetration in L-band dataset it is also expected to obtain more coherent interferograms due to the reduced impact of atmospheric perturbation or changes in ground surface conditions.

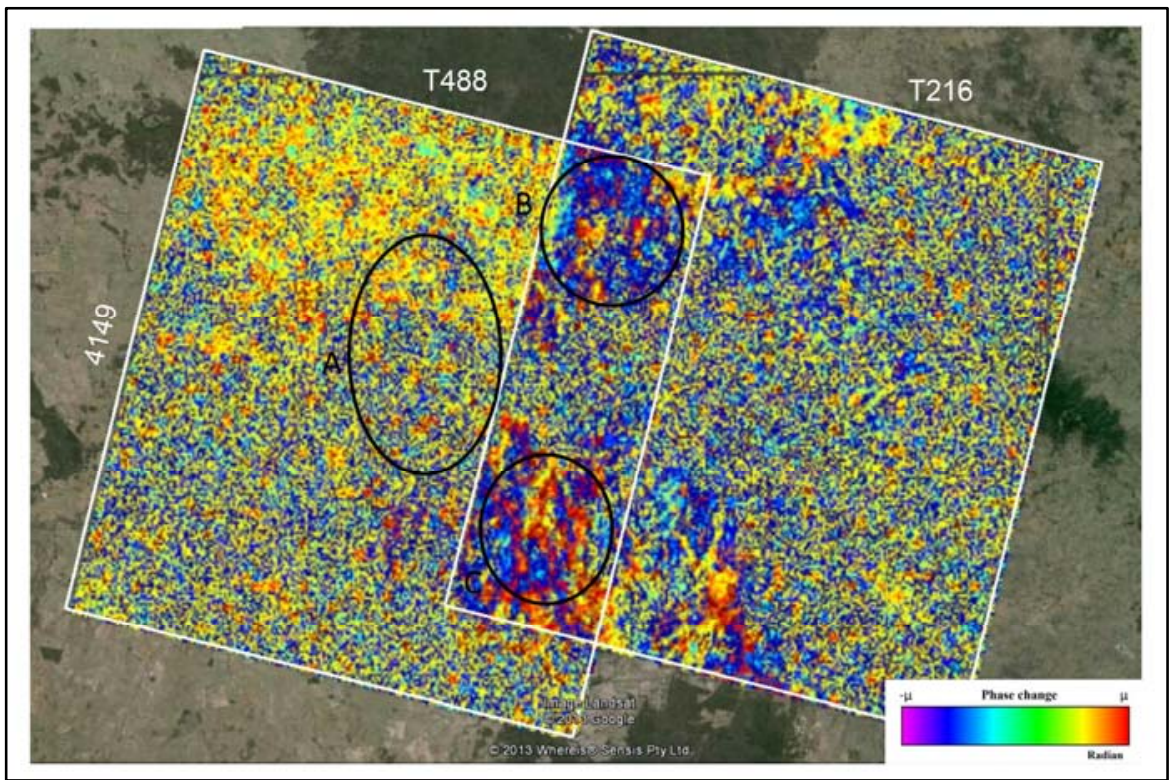

Figure 4. Preliminary DInSAR processing results of ERS-1 (19921995) in descending path. The areas B and C indicate deformation signals in the monitoring time interval.

\subsection{DInSAR signals and Geological - Geophysical Perspective}

To build a subsurface structural model and train the proposed algorithm for simulation, geological and geophysical information over areas with deformation signals needs to be obtained. The most common geological formations in this area are sandstone, mudstone of the Kumbarilla beds formed during the Jurassic to Cretaceous age, siltstone, mudstone and quartos of the Bungil formation, as well as flood plain alluvium of Quaternary age, and conglomerates of chinchilla sand. The yellow circles in Figure 6 highlight Envisat ASAR signals, while the orange stars represent locations detected by RADARSAT-2. 


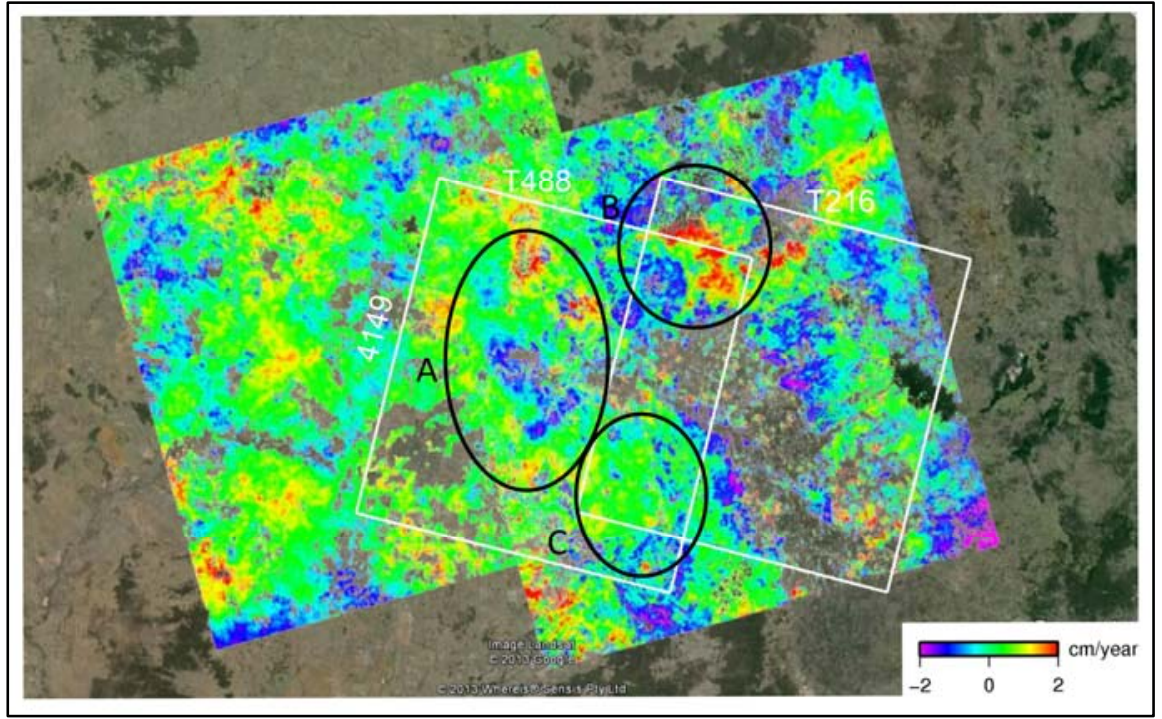

Figure 5. Preliminary DInSAR processing of RADARSAT-2 (20122013) in ascending path overlaid by Envisat ASAR descending footprints in white outlines. The deformation signals are in $\mathrm{A}, \mathrm{B}$, and $\mathrm{C}$ areas compatible with the previous Envisat observation in similar regions.

From geophysical point of view two major fault lines spatially confine the signals in dashed area and force them to follow a specific distribution pattern. DInSAR observations show that the deformation signals' distribution is generally random but when fault lines are in place, subsidence patterns appear to follow the confining structures as discussed in (Amelung et al.). In combination with the large number of seismic lines obtained since the 1960s (red and black lines) and covering the significant parts of the basin, more detailed information should be obtainable from merging those two types of information. For example, the red lines are seismic acquisitions which could be used in structural model development along with the groundwater extraction wells (blue dots), and other seismic lines that will be processed for further analysis (black lines).

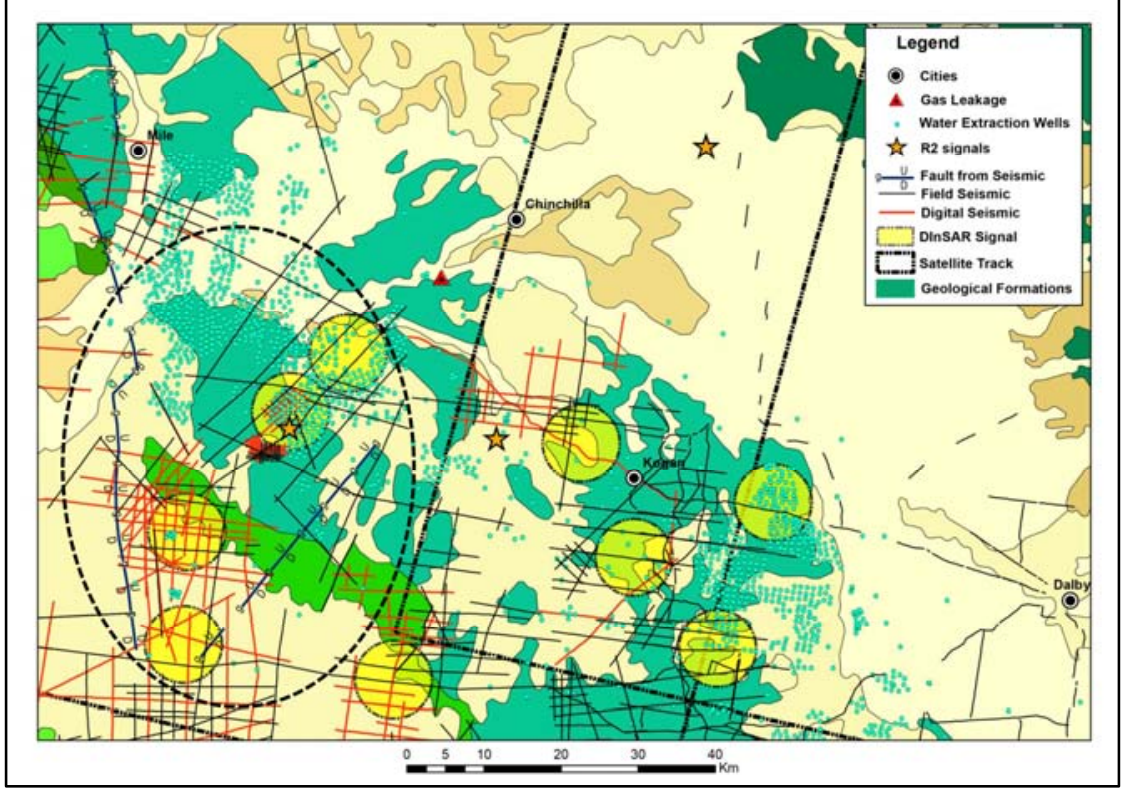

Figure 6. DInSAR signals within the Surat basin overlaid on geological and geophysical data with groundwater extraction sites.

\section{MODEL SCOPE}

In order to retrieve subsurface geophysical parameters such as size and depth of source from satellite observations, inverse geophysical modeling approaches are generally applied. The methods make use of the mean velocity deformation measurements obtained from the DInSAR time series analysis as an input to characterize the source parameters which are embedded in an elastic homogeneous half-space (Hooper \& Wright, 2007). The most common analytical source models that fit the surface deformations are the point pressure source (Mogi, 1958), ellipsoidal source (Yang et al., 1988) and disk aquifer models (Segall, 1992). Other inversion algorithms which are used for volcanic sources and subsidence are the Penny shaped crack model (Fialko et al., 2001), and also finite element modeling for a multi-layered earth considering its rheology (De Zeeuw-van Dalfsen et al., 2012).Among these different approaches for the estimation of depth and thickness of an aquifer, the disk aquifer model, dealing with the poroelastic nature of source, could be an option for simulation. However, appropriate poroelastic constants, reservoir-wide stress change, compressional and shear velocities are required, which is rarely the case. For the modeling part of this study, a disk aquifer model (Segall, 1992) will be used to solve the inverse problem, i.e. to define the parameter space of the structural properties (stress, strain, porosity and permeability, etc.) underneath the deformed area,. In the case of either subsurface ground water extraction or shrinkage/swelling of clayey soils in the upper layers, more 
flexible data-driven algorithms such as a Neural Network could be useful (Stramondo et al., 2011). Neural Networks efficiently include information from independent sources as input vectors for best optimization within the geophysical modeling framework. In this context, the aim is to use Neural Networks to improve the source parameterization by integrating DInSAR displacement velocity observations and other ground based geological and geophysical information (Figure 7). This approach is composed of a number of nonlinear elements, linked together through connections defined by weighting factors. Such a structure makes neural networks inherently suitable for addressing nonlinear problems and improving retrieval of structural properties within the Surat Basin. Figure 7 outlines a general overview for the framework to extract and modify subsurface information. ground-based observations such as surface geology, seismic interpretation and soil sampling along with airborne(electromagnetic surveys) and space borne( DInSAR observations) would be used as network training dataset to extract parameters like porosity, permeability and compressibility which were used to be obtained by geophysical surveys. For this framework around 70 percent of the available datasets are treated as training data and the remaining 30 percent will be used for model validation and verification. Training and testing of this network will be performed for three or four different scenarios to define the best structural parameters.

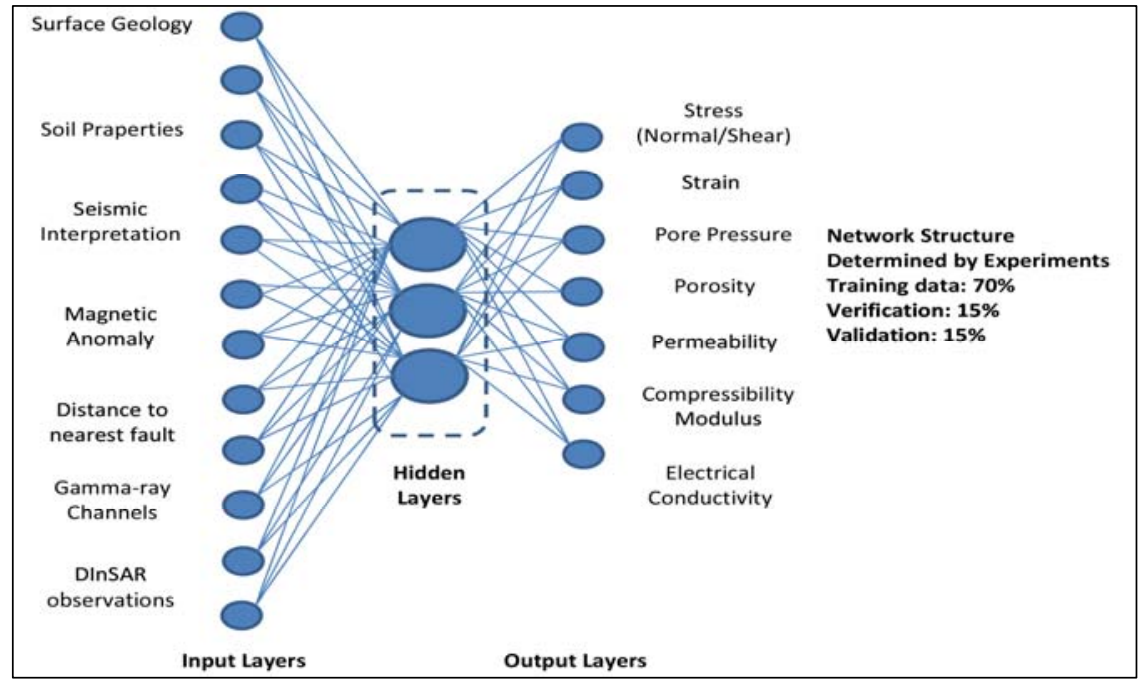

Figure 7.General overview for neural network scheme to be used for subsurface structural modeling

\section{DISCUSSION AND CONCLUSIONS}

Geophysical parameters are required to adequately address issues arising during the planning stage of large scale groundwater extraction. To collect accurate information of subsurface features even at the local scale, labor intensive and time consuming processes are inevitable. The interpretation of such in-situ observations is sometimes complex and inaccurate. Consequently, there is a need for large scale and more accurate data collection strategies. In this paper an alternative approach for seismic or bore hole data to retrieve subsurface parameters and to improve the structural model is introduced, by using DInSAR surface deformation maps derived from different satellite platforms across the Surat Basin. It is proposed to use such surface deformation information to improve the geophysical models through geophysical inverse modeling.

The results presented in this paper have underlined that DInSAR observations are indeed reliable sources of deformation signals within the Surat Basin. For a detailed analysis, ERS-1 and RADARSAT-2 imagery were studied for the years 1992-1995 and 2012-2013. The ERS-1 deformation maps show clear signals in the proposed area which re-appeared in the 2012-2013 observations of RADARSAT-2. These patterns in two different times confirm the gradual and continuous deformation in this particular region between Chinchilla and Dalby. Based on the assumption that such data is a reflection of the subsurface structure and extractions, it will be possible to derive the relevant subsurface parameters. For the long-term monitoring perspective of the Surat Basin, both L-band and C-band datasets will be jointly used to develop more accurate observations. The resulted mean velocity deformation measurements of DInSAR monitoring will then be used as an input for analytical inverse modeling to maintain the structural properties of the deformation source. Improving the available subsurface structural model from in-situ geological and geophysical observations is the following stage could be achieved by training Neural Network through simulated and in-situ structural properties.

\section{ACKNOWLEDGMENTS}

The authors would like to thank the European Space Agency and the Japan Aerospace Exploration Agency (JAXA) for the provision of satellite datasets in C- (ESA PI. 13196) and L-band (JAXA PI-1338), respectively. RADARSAT-2 data were made available through the Canadian Space Agency. For support in 
the processing of C-band dataset and the SARPROZ software we gratefully acknowledge Prof. Perissin at the Chinese University of Hong Kong (CUHK). Finally, the Geoscience Australia InSAR team, Queensland Government, and 3D-Geo Pty Ltd in Melbourne made the seismic in-situ measurement and analysis available for this study.

\section{REFERENCES}

Amelung, F., et al.(1999). Sensing the ups and downs of Las Vegas: InSAR reveals structural control of land subsidence and aquifer-system deformation. Geology, 27(6),483-486.

Anderssohn, J., et al.(2009). Surface deformation time series and source modelling for a volcanic complex system based on satellite wide swath and image mode interferometry: The Lazufre system, central Andes. Remote Sensing of Environmen,113(10), 2062-2075.

Berardino, P., Fornaro, G., Lanari, R., and Sansosti, E. (2002). A new algorithm for surface deformation monitoring based on small baseline differential SAR interferograms. IEEE Transaction on Geoscience and Remote Sensing, 40, 2375-2383.

De Zeeuw-van Dalfsen, E., Pedersen, R., Hooper, A., and Sigmundsson, F.(2012). Subsidence of Askja caldera 2000-2009: Modelling of deformation processes at an extensional plate boundary, constrained by time series InSAR analysis. Journal of Volcanology and Geothermal Research, 213-214, 72-82.

Fialko,Y., Khazan, Y., and Simons, M. (2001). Deformation due to a pressurized horizontal circular crack in an elastic half-space, with applications to volcano geodesy. Geophysical Journal International, 146(1), 181-190.

Hooper, A. and Wright, T.J. (2007). Comparison of Monte Carlo methods for model probability distribution determination in SAR interferometry. Proceedings Fringe Workshop 2010. Frascati2010, Italy.

Massonnet, D., and Feigl, K.L. (1998). Radar interferometry and its application to changes in the earth's surface. Reviews of Geophysics, 36, 441-500.

Mogi, K.(1958). Relations between the eruptions of various volcanoes and the deformations of the ground surface around them. Bull. Earthquake Res.Inst.Univ.Tokyo, 36, 99-134.

Moran, C. \& Vink, S.(2010). Assessment of impacts of the proposed coal seam gas operations on surface and groundwater systems in the Murray-Darling Basin. Centre for Water in the Mineral Industry, Sustainable Minerals Institute, The University of Queensland.

Perissin, D., Wang, Z., and Wang, T. (2012). The SARPROZ InSAR tool for urban subsidence/manmade structure stability monitoring in China.

Queensland Water Commission(2012). Surat underground water impact report-Department of Natural Resources and Mines.

Rosen, P.A., Hensley, S., Joughin, I.R., Li, F.K., Madsen, S.N., Rodriguez, E., and Goldstein, R.M. (2000). Synthetic aperture radar interferometry. Proceedings of the IEEE, 88, 333-382.

Rohmer, J., and Raucoules, D. (2012). On the applicability of Persistent Scatterers Interferometry (PSI) analysis for long term CO2 storage monitoring. Engineering Geology, 147-148, 137-148.

Samsonov, S. and d'Oreye, N. (2012). Multidimensional time-series analysis of ground deformation from multiple InSAR datasets applied to Virunga Volcanic Province. Geophysical Journal International, 191(3). 1095-1108.

Segall, P., (1992) Induced stresses due to fluid extraction from axisymmetric reservoirs, Pure \& Applied Geophysics, 139(3-4),535-560.

Stramondo, S., et al.,(2011). Seismic source parameters from InSAR data through neural networks, IEEE Transaction on Geoscience and Remote Sensing, 49(1), 96-104.

Yang, X.M., Davis, P.M., and Dieterich, J.H.(1988). Deformation from inflation of a dipping finite prolate spheroid in an elastic half-space as a model for volcanic stressing. Journal of Geophysical Research, 93(B5), 4249-4257.

Zhang, H.(2010), An integrated approach to determine parameters of a 3D volcano model by using InSAR data with metamodel technique, IEEE Geoscience and Remote Sensing Symposium (IGARSS), 1648-165 